K. Saranya, V.Piramanantham, and E. Thandapani

\title{
Oscillation Results for Third-Order Semi-Canonical Quasi-Linear Delay Differential Equations
}

https://doi.org/10.1515/msds-2020-0135

Received June 26, 2021; accepted October 13, 2021

Abstract: The main purpose of this paper is to study the oscillatory properties of solutions of the third-order quasi-linear delay differential equation

$$
\mathcal{L} y(t)+f(t) y^{\beta}(\sigma(t))=0
$$

where $\mathcal{L} y(t)=\left(b(t)\left(a(t)\left(y^{\prime}(t)\right)^{\alpha}\right)^{\prime}\right)^{\prime}$ is a semi-canonical differential operator. The main idea is to transform the semi-canonical operator into canonical form and then obtain new oscillation results for the studied equation. Examples are provided to illustrate the importance of the main results.

Keywords: Semi- canonical, third-order, delay differential equations, oscillation

MSC: 34C10, 34K11

\section{Introduction}

In this paper, we are concerned with the third-order quasi-linear delay differential equation

$$
\mathcal{L} y(t)+f(t) y^{\beta}(\sigma(t))=0, t \geq t_{0}>0,
$$

where $\mathcal{L}$ denote the differential operator $\mathcal{L} y(t)=\left(b(t)\left(a(t)\left(y^{\prime}(t)\right)^{\alpha}\right)^{\prime}\right)^{\prime}$. Throughout, we will assume that:

$\left(\mathrm{H}_{1}\right) a \in C^{(2)}\left(\left[t_{0}, \infty\right)\right), b \in C^{1}\left(\left[t_{0}, \infty\right)\right), a(t)>0$ and $b(t)>0$ for all $t \geq t_{0}$;

$\left(\mathrm{H}_{2}\right) f(t) \in C\left(\left[t_{0}, \infty\right)\right)$ is a positive for all $t \geq t_{0}$;

$\left(\mathrm{H}_{3}\right) \sigma(t) \in C^{1}\left(\left[t_{0}, \infty\right)\right), \sigma(t)<t, \lim _{t \rightarrow \infty} \sigma(t)=\infty$ and $\sigma^{\prime}(t) \geq 0$ for all $t \geq t_{0}$;

$\left(\mathrm{H}_{4}\right) \alpha$ and $\beta$ are ratios of odd positive integers;

$\left(\mathrm{H}_{5}\right)$ The operator $\mathcal{L}$ is in semi-canonical form, that is,

$$
\Pi\left(t_{0}\right)=\int_{t_{0}}^{\infty} \frac{1}{b(t)} d t<\infty \text { and } \int_{t_{0}}^{\infty} \frac{1}{a^{\frac{1}{\alpha}}(t)} d t=\infty .
$$

Under the solution of (1.1), we mean a nontrivial function $y(t) \in C\left(\left[t_{y}, \infty\right)\right), t_{y} \geq t_{0}$ which has the properties $y(t) \in C^{1}\left(\left[t_{y}, \infty\right)\right), t_{y} \geq t_{0}, a(t)\left(y^{\prime}(t)\right)^{\alpha} \in C^{1}\left(\left[t_{y}, \infty\right)\right), b(t)\left(a(t)\left(y^{\prime}(t)\right)^{\alpha}\right)^{\prime} \in C^{1}\left(\left[t_{y}, \infty\right)\right), t_{y} \geq t_{0}$ and satisfies (1.1) on $\left[t_{y}, \infty\right)$. Our attention is restricted to those solutions $y(t)$ of (1.1) satisfying $\sup \{|y(t)|: t \geq T\}>0$ for all

K. Saranya, V.Piramanantham: Department of Mathematics, Bharathidasan University, Tiruchirappalli-620024, India, E-mail: saranyakarunamurthy@gmail.com,vpm@bdu.ac.in

E. Thandapani: Ramanujan Institute for Advanced Study in Mathematics, University of Madras, Chennai-600005, India, E-mail: ethandapani@yahoo.co.in 
$T \geq t_{y}$. We assume that (1.1) possesses such a solution. A solution of (1.1) is called oscillatory if it has infinitely many zeros in $\left[t_{y}, \infty\right)$; otherwise it is termed as nonoscillatory. Equation (1.1) is said to be oscillatory if all its solutions are oscillatory.

In recent years, a great interest has been devoted to study the oscillatory behavior of solutions of thirdorder differential equations, see [1-10, 12-16, 18-21, 24, 25, 27, 29, 30, 32, 34]. Since this is very helpful in predicting the similar behavior of solutions of third-order partial differential equations [22, 26, 33].

From the review of literature, it is clear that most of the papers are dealing with the oscillatory and asymptotic behavior of solutions of (1.1) under the canonical type condition

$$
\int_{t_{0}}^{\infty} \frac{1}{b(t)} d t=\infty \text { and } \int_{t_{0}}^{\infty} \frac{1}{a^{\frac{1}{\alpha}}(t)} d t=\infty,
$$

see, for example $[1,2,5-7,9,13-16,18,21,29,30,32,34]$ and the references cited therein. This is due to the fact that the examination of canonical type equation is much simpler than non-canonical equations. Therefore in this paper we first introduce a technique that connect the semi-canonical equation (1.1) with that of canonical type equation and then we examine the behavior of its solutions.

While considering nonoscillatory solutions of (1.1), we can restrict our attention to positive ones. From the well-known results in $[17,23,27]$, it follows that the set of positive solutions of (1.1) has the following structure:

Lemma 1.1. Assume that $y(t)$ is an eventually positive solution of (1.1), then $y(t)$ satisfies one of the following three options:

(I) $y(t)>0,\left(y^{\prime}(t)\right)^{\alpha}>0,\left(a(t)\left(y^{\prime}(t)\right)^{\alpha}\right)^{\prime}>0,\left(b(t)\left(a(t)\left(y^{\prime}(t)\right)^{\alpha}\right)^{\prime}\right)^{\prime}<0$,

(II) $y(t)>0,\left(y^{\prime}(t)\right)^{\alpha}<0,\left(a(t)\left(y^{\prime}(t)\right)^{\alpha}\right)^{\prime}>0,\left(b(t)\left(a(t)\left(y^{\prime}(t)\right)^{\alpha}\right)^{\prime}\right)^{\prime}<0$,

(III) $y(t)>0,\left(y^{\prime}(t)\right)^{\alpha}>0,\left(a(t)\left(y^{\prime}(t)\right)^{\alpha}\right)^{\prime}<0,\left(b(t)\left(a(t)\left(y^{\prime}(t)\right)^{\alpha}\right)^{\prime}\right)^{\prime}<0$,

eventually for all sufficiently large $t$.

So, if we want to obtain oscillation criteria for semi-canonical equation (1.1), we have to eliminate three above mentioned cases, which may lead to three conditions. To overcome this, we assume a simple condition that yields to a canonical form and this essentially simplifies the examination of (1.1).

\section{Main Results}

Throughout the paper, we employ the following notations:

$$
\begin{aligned}
& \Pi(t)=\int_{t}^{\infty} \frac{1}{b(t)} d t, \quad d(t)=b(t) \Pi^{2}(t), \quad c(t)=\frac{a(t)}{\Pi(t)}, \\
& F(t)=\Pi(t) f(t), \quad G(t)=F(t)(\eta(\sigma(t)))^{\alpha}, \\
& \mu(t)=\int_{t_{1}}^{t} \frac{1}{d(s)} d s \quad \text { and } \quad \eta(t)=\int_{t_{1}}^{t}\left(\frac{\mu(s)}{c(s)}\right)^{1 / \alpha} d s
\end{aligned}
$$

for all $t \geq t_{1}$, where $t_{1} \geq t_{0}$.

Theorem 2.1. If

$$
\int_{t_{0}}^{\infty}\left(\frac{\Pi(t)}{a(t)}\right)^{1 / \alpha} d t=\infty
$$


then the semi-canonical operator $\mathcal{L} y$ has the following unique canonical representation

$$
\mathcal{L} y(t)=\frac{1}{\Pi(t)}\left(b(t) \Pi^{2}(t)\left(\frac{a(t)}{\Pi(t)}\left(y^{\prime}(t)\right)^{\alpha}\right)\right)^{\prime} .
$$

Proof. Direct calculation shows that

$$
b(t) \Pi^{2}(t)\left(\frac{a(t)}{\Pi(t)}\left(y^{\prime}(t)\right)^{\alpha}\right)^{\prime}=\Pi(t) b(t)\left(a(t)\left(y^{\prime}(t)\right)^{\alpha}\right)^{\prime}+a(t)\left(y^{\prime}(t)\right)^{\alpha} .
$$

So

$$
\left(b(t) \Pi^{2}(t)\left(\frac{a(t)}{\Pi(t)}\left(y^{\prime}(t)\right)^{\alpha}\right)^{\prime}\right)^{\prime}=\Pi(t)\left(b(t)\left(a(t)\left(y^{\prime}(t)\right)^{\alpha}\right)^{\prime}\right)^{\prime}
$$

or

$$
\frac{1}{\Pi(t)}\left(b(t) \Pi^{2}(t)\left(\frac{a(t)}{\Pi(t)}\left(y^{\prime}(t)\right)^{\alpha}\right)^{\prime}\right)^{\prime}=\left(b(t)\left(a(t)\left(y^{\prime}(t)\right)^{\alpha}\right)^{\prime}\right)^{\prime} .
$$

This shows that (2.2) is in the canonical form, that is,

$$
\int_{t_{0}}^{\infty} \frac{1}{b(t) \Pi^{2}(t)} d t=\int_{t_{0}}^{\infty} d\left(\frac{1}{\Pi(t)}\right)=\lim _{t \rightarrow \infty} \frac{1}{\Pi(t)}=\frac{1}{\Pi\left(t_{0}\right)}=\infty,
$$

and

$$
\int_{t_{0}}^{\infty}\left(\frac{\Pi(t)}{a(t)}\right)^{1 / \alpha} d t=\infty
$$

by (2.1). However Trench proved in [32] that there exists the only one canonical representation of $\mathcal{L}$ (up to multiplicative constants with product 1) and so our canonical form is unique. This completes the proof.

Now it follows from Theorem 2.1 that (1.1) can be written in the canonical form as

$$
\left(d(t)\left(c(t)\left(y^{\prime}(t)\right)^{\alpha}\right)^{\prime}\right)^{\prime}+F(t) y^{\beta}(\sigma(t))=0,
$$

and the following result is immediate.

Theorem 2.2. Assume (2.1) holds. Semi-canonical equation (1.1) possesses solution $y(t)$ if and only if the canonical equation (2.3) has the solution $y(t)$.

Corollary 2.3. Assume that (2.1) holds. Semi-canonical equation (1.1) has an eventually positive solution if and only if the canonical equation (2.3) has an eventually positive solution.

Corollary 2.3 significantly simplifies examination of (1.1) since for (2.3) we deal with only two classes of an eventually positive solutions (see, [[24], Lemma 2]), namely either

$$
y(t)>0, c(t)\left(y^{\prime}(t)\right)^{\alpha}<0, d(t)\left(c(t)\left(y^{\prime}(t)\right)^{\alpha}\right)^{\prime}>0,\left(d(t)\left(c(t)\left(y^{\prime}(t)\right)^{\alpha}\right)^{\prime}\right)^{\prime}<0,
$$

and in this case we say $y \in \mathcal{N}_{0}$ or

$$
y(t)>0, c(t)\left(y^{\prime}(t)\right)^{\alpha}>0, d(t)\left(c(t)\left(y^{\prime}(t)\right)^{\alpha}\right)^{\prime}>0,\left(d(t)\left(c(t)\left(y^{\prime}(t)\right)^{\alpha}\right)^{\prime}\right)^{\prime}<0,
$$

and in this case we denote that $y \in \mathcal{N}_{2}$.

Lemma 2.4. Assume that (2.3) possesses an eventually positive solution $y(t) \in \mathcal{N}_{2}$. Then

$$
y(t) \geq \eta(t)\left(d(t)\left(c(t)\left(y^{\prime}(t)^{\alpha}\right)^{\prime}\right)\right)^{1 / \alpha},
$$

and

$$
\frac{c(t)\left(y^{\prime}(t)\right)^{\alpha}}{\mu(t)} \text { is decreasing. }
$$


Proof. The proof is similar to that of [13, Lemma 2] and [8, Lemma 3.1] and therefore skipped.

Our next lemma is a particular case $(n=1, m=1)$ of Corollary 1 of Philos [28].

Lemma 2.5. Let $f \in C\left(\left[t_{0}, \infty\right) \times R, R\right), \sigma \in\left(\left[t_{0}, \infty\right), R\right), f(t, x) \operatorname{sgn} x=\operatorname{sgn} x, t \geq t_{0}, \lim _{t \rightarrow \infty} \sigma(t)=\infty$ and $\sigma(t)<t$ for every $t \geq t_{0}$. If $y$ is a positive bounded solution of the differential inequality

$$
y^{\prime}(t)+f(t, y(\sigma(t))) \leq 0, t \geq t_{0}
$$

then there exists a positive solution $x$ of the differential equation

$$
x^{\prime}(t)+f(t, x(\sigma(t)))=0
$$

such that $x(t) \leq y(t)$ for all large $t$, and $\lim _{t \rightarrow \infty} x(t)=0$ monotonically.

In the remaining part of the paper, we always assume condition (2.1) holds without further mention.

Theorem 2.6. If $\alpha=\beta$ and

$$
\limsup _{t \rightarrow \infty} \int_{\sigma(t)}^{t}\left(c(s) \int_{s}^{t} \frac{1}{d(u)} \int_{u}^{t} F(v) d v d u\right)^{1 / \alpha} d s>1,
$$

then every positive solution $y(t)$ of (1.1) does not satisfy $\mathcal{N}_{0}$.

Proof. Assume that $y(t)$ is an eventually positive solution of (1.1). By Corollary 2.3, the function $y(t)$ is a positive solution of (2.3). Now assume to the contrary that $y(t) \in \mathcal{N}_{0}$. Integrating (2.3) from $s$ to $t$ yields

$$
d(s)\left(c(s)\left(y^{\prime}(s)\right)^{\alpha}\right)^{\prime} \geq \int_{s}^{t} F(v) y^{\beta}(\sigma(v)) d v \geq y^{\beta}(\sigma(t)) \int_{s}^{t} F(v) d v .
$$

Again integrating twice from $s$ to $t$, one gets

$$
y(s) \geq y^{\beta / \alpha}(\sigma(t)) \int_{s}^{t}\left(\frac{1}{c(j)} \int_{j}^{t} \frac{1}{d(u)} \int_{u}^{t} F(v) d v d u\right)^{1 / \alpha} d j .
$$

By setting $s=\sigma(t)$ and $\alpha=\beta$, we obtain a contradiction to (2.6). This completes the proof.

Theorem 2.7. If $\alpha=\beta$ and

$$
\liminf _{t \rightarrow \infty} \int_{\sigma(t)}^{t} G(s) d s>1 / e,
$$

then every positive solution $y(t)$ of (1.1) does not satisfy $\mathcal{N}_{2}$.

Proof. Let $y(t)$ be an eventually positive solution of (1.1). Then by Corollary 2.3, the function $y(t)$ is also a positive solution of (2.3). Now assume to the contrary that $y(t) \in \mathcal{N}_{2}$. Since $d(t)\left(c(t)\left(y^{\prime}(t)\right)^{\alpha}\right)^{\prime}$ is positive and decreasing, we can verify that

$$
\begin{aligned}
c(t)\left(y^{\prime}(t)\right)^{\alpha} & \geq \int_{t_{1}}^{t} \frac{\left.d(s) c(s)\left(y^{\prime}(s)\right)^{\alpha}\right)^{\prime}}{d(s)} d s \\
& \geq d(t)\left(c(t)\left(y^{\prime}(t)\right)^{\alpha}\right)^{\prime} \int_{t_{1}}^{t} \frac{1}{d(s)} d s .
\end{aligned}
$$


Integrating again from $t_{1}$ to $t$ yields

$$
y(t) \geq\left(d(t)\left(c(t)\left(y^{\prime}(t)\right)^{\alpha}\right)^{\prime}\right)^{1 / \alpha} \int_{t_{1}}^{t}\left(\frac{1}{c(s)} \int_{t_{1}}^{s} \frac{1}{d(u)} d u\right)^{1 / \alpha} d s .
$$

Using this in (2.3), we see that $w(t)=d(t)\left(c(t)\left(y^{\prime}(t)\right)^{\alpha}\right)^{\prime}$ is a positive solution of the differential inequality

$$
w^{\prime}(t)+F(t)\left(\int_{t_{1}}^{\sigma(t)}\left(\frac{1}{c(s)} \int_{t_{1}}^{s} \frac{1}{d(u)} d u\right)^{1 / \alpha} d s\right)^{\alpha} w(\sigma(t)) \leq 0 .
$$

This is a contradiction since by Theorem 2.1.1 in [17], condition (2.7) guarantees that (2.8) has no positive solution. This completes the proof.

Combining Theorems 2.6 and 2.7, one can immediately obtain the following oscillation result:

Theorem 2.8. Let $\alpha=\beta$, (2.6) and (2.7) hold. Then (1.1) is oscillatory.

Next we consider the case $\alpha>\beta$.

Theorem 2.9. Let $\alpha>\beta$ holds. If

$$
\limsup _{t \rightarrow \infty} \int_{\sigma(t)}^{t}\left(\frac{1}{c(s)} \int_{s}^{t} \frac{1}{d(u)} \int_{u}^{t} F(v) d v d u\right)^{1 / \alpha} d s=\infty
$$

and

$$
\int_{t_{1}}^{\infty} F(t)(\eta(\sigma(t)))^{\beta} d t=\infty
$$

for all $t_{1} \geq t_{0}$, then (1.1) is oscillatory.

Proof. Let $y(t)$ be an eventually positive solution of (1.1). It follows from Corollary 2.3 that $y(t)$ is also a positive solution of (2.3) and either $y(t) \in \mathcal{N}_{0}$ or $y(t) \in \mathcal{N}_{2}$.

First assume that $y(t) \in \mathcal{N}_{0}$. Then proceeding as in the proof of Theorem 2.6, we obtain

$$
y(\sigma(t)) \geq y^{\beta / \alpha}(\sigma(t)) \int_{\sigma(t)}^{t}\left(\frac{1}{c(s)} \int_{s}^{t} \frac{1}{d(u)} \int_{u}^{t} F(v) d v d u\right)^{1 / \alpha} d s
$$

or

$$
y^{1-\beta / \alpha}(\sigma(t)) \geq \int_{\sigma(t)}^{t}\left(\frac{1}{c(s)} \int_{s}^{t} \frac{1}{d(u)} \int_{u}^{t} F(v) d v d u\right)^{1 / \alpha} d s .
$$

Since $y(t)$ is decreasing and $\alpha>\beta$, we see that $y^{1-\beta / \alpha}(\sigma(t)) \leq M$ for all $t \geq t_{1} \geq t_{0}$. using this, we obtain

$$
M \geq \int_{\sigma(t)}^{t}\left(\frac{1}{c(s)} \int_{s}^{t} \frac{1}{d(u)} \int_{u}^{t} F(v) d v d u\right)^{1 / \alpha} d s,
$$

which contradicts (2.9) as $t \rightarrow \infty$.

Next assume that $y(t) \in \mathcal{N}_{2}$. Let $w(t)=d(t)\left(c(t)(y(t))^{\alpha}\right)^{\prime}>0$. Then using (2.4) in (2.3), we obtain

$$
w^{\prime}(t)+\eta^{\beta}(\sigma(t)) F(t)(w(\sigma(t)))^{\beta / \alpha} \leq 0, t \geq t_{1} .
$$


Since $w(t)$ is a positive bounded solution of the last inequality then by Lemma 2.5, we see that the corresponding equation

$$
w^{\prime}(t)+\eta^{\beta}(\sigma(t)) F(t)(w(\sigma(t)))^{\beta / \alpha}=0, t \geq t_{1},
$$

has also a positive solution. But by Theorem 3.9.3 of [17], the condition (2.10) implies that $w(t)$ is oscillatory. This contradiction completes the proof of the theorem.

Theorem 2.10. Assume that there exists a function $\zeta(t) \in C^{\prime}\left(\left[t_{0}, \infty\right)\right)$ such that

$$
\zeta^{\prime}(t) \geq 0, \zeta(t)>t, \tau(t)=\sigma(\zeta(\zeta(t)))<t .
$$

If for all sufficiently large $t_{1} \geq t_{0}$, the first order delay differential equations

$$
w^{\prime}(t)+Q_{1}(t) w^{\beta / \alpha}(\sigma(t))=0
$$

and

$$
w^{\prime}(t)+Q_{2}(t) w^{\beta / \alpha}(\tau(t))=0
$$

where

$$
\begin{gathered}
Q_{1}(t)=F(t)\left(\int_{t_{1}}^{\sigma(t)}\left(\frac{1}{c(s)} \int_{t_{1}}^{s} \frac{1}{d(u)} d u\right)^{1 / \alpha} d s\right)^{\beta} \\
Q_{2}(t)=\left(\frac{1}{c(t)} \int_{t}^{\zeta(t)} \frac{1}{d(s)} \int_{s}^{\zeta(s)} F(u) d u d s\right)^{1 / \alpha}
\end{gathered}
$$

are oscillatory, then (1.1) is oscillatory.

Proof. Let $y(t)$ be an eventually positive solution of (1.1). It follows from Corollary 2.3 that $y(t)$ is also a positive solution of (2.3) and either $y(t) \in \mathcal{N}_{0}$ or $y(t) \in \mathcal{N}_{2}$.

If $y(t) \in \mathcal{N}_{2}$, then by using the fact that

$$
w(t)=d(t)\left(c(t)\left(y^{\prime}(t)\right)^{\alpha}\right)^{\prime}>0
$$

is decreasing, we have

$$
c(t)\left(y^{\prime}(t)\right)^{\alpha} \geq \int_{t_{1}}^{t} \frac{1}{d(s)}\left(d(s)\left(c(s)\left(y^{\prime}(s)\right)^{\alpha}\right)^{\prime}\right) d s \geq w(t) \int_{t_{1}}^{t} \frac{1}{d(s)} d s .
$$

Integrating from $t_{1}$ to $t$, we are led to

$$
\begin{aligned}
y(t) & \geq \int_{t_{1}}^{t}\left(\frac{w(s)}{c(s)} \int_{t_{1}}^{s} \frac{1}{d(u)} d u\right)^{1 / \alpha} d s \\
& \geq w^{1 / \alpha}(t) \int_{t_{1}}^{t}\left(\frac{1}{c(s)} \int_{t_{1}}^{s} \frac{1}{d(u)} d u\right)^{1 / \alpha} d s .
\end{aligned}
$$

Hence

$$
y(\sigma(t)) \geq w^{1 / \alpha}(\sigma(t)) \int_{t_{1}}^{\sigma(t)}\left(\frac{1}{c(s)} \int_{t_{1}}^{s} \frac{1}{d(u)} d u\right)^{1 / \alpha} d s .
$$

Combining the last inequality together with (2.3), we obtain

$$
-w^{\prime}(t) \geq F(t)\left(\int_{t_{1}}^{\sigma(t)}\left(\frac{1}{c(s)} \int_{t_{1}}^{s} \frac{1}{d(u)} d u\right)^{\frac{1}{\alpha}} d s\right)^{\beta} w^{\beta / \alpha}(\sigma(t)) .
$$


Therefore, it is clear that $w(t)$ is a positive solution of differential inequality

$$
w^{\prime}(t)+Q_{1}(t) w^{\beta / \alpha}(\sigma(t)) \leq 0
$$

for $t \geq t_{1}$. Since $w(t)$ is a positive bounded solution of the last inequality and therefore by Lemma 2.5 , we conclude that there exists a positive solution $w(t)$ of equation (2.12) with $\lim _{t \rightarrow \infty} w(t)=0$, which contradicts the fact that equation (2.12) is oscillatory.

Next, we assume that $y(t) \in \mathcal{N}_{0}$. An integration of (2.3) from $t$ to $\zeta(t)$ yields

$$
\begin{aligned}
d(t)\left(c(t)\left(y^{\prime}(t)\right)^{\alpha}\right)^{\prime} & \geq \int_{t}^{\zeta(t)} F(s) y^{\beta}(\sigma(s)) d s \\
& \geq y^{\beta}(\sigma(\zeta(t))) \int_{t}^{\zeta(t)} F(s) d s .
\end{aligned}
$$

Then

$$
\left(c(t)\left(y^{\prime}(t)\right)^{\alpha}\right)^{\prime} \geq \frac{y^{\beta}(\sigma(\zeta(t)))}{d(t)} \int_{t}^{\zeta(t)} F(s) d s
$$

Integrating the last inequality from $t$ to $\zeta(t)$, we have

$$
\begin{aligned}
-c(t)\left(y^{\prime}(t)\right)^{\alpha} & \geq \int_{t}^{\zeta(t)} \frac{y^{\beta}(\sigma(\zeta(s)))}{d(s)} \int_{s}^{\zeta(s)} F(u) d u d s \\
& \geq y^{\beta}(\tau(t)) \int_{t}^{\zeta(t)} \frac{1}{d(s)} \int_{s}^{\zeta(s)} F(u) d u d s .
\end{aligned}
$$

Dividing the last inequality by $c(t)$ and then integrating it from $t$ to $\infty$, one gets

$$
y(t) \geq \int_{t}^{\infty}\left(\frac{y^{\beta}(\tau(s))}{c(s)} \int_{s}^{\zeta(s)} \frac{1}{d(u)} \int_{u}^{\zeta(u)} F(v) d v d u\right)^{1 / \alpha} d s .
$$

Set the right-hand side of the last inequality by $w(t)$. Then $y(t) \geq w(t)>0$ and it is easy to verify that

$$
\begin{aligned}
0 & =w^{\prime}(t)+\left(\frac{1}{c(t)} \int_{t}^{\zeta(t)} \frac{1}{d(s)} \int_{s}^{\zeta(s)} F(u) d u d s\right)^{1 / \alpha} d s y^{\beta / \alpha}(\tau(t)) \\
& \geq w^{\prime}(t)+Q_{2}(t) w^{\beta / \alpha}(\tau(t)) .
\end{aligned}
$$

Since $w(t)$ is a positive bounded solution of the last inequality, then by Lemma 2.5 we see that the corresponding differential equation (2.13) has also a positive solution with $\lim _{t \rightarrow \infty} w(t)=0$. This contradicts the assumption that (2.13) is oscillatory, and hence we conclude that (1.1) oscillates. This completes the proof of the theorem.

Employing criteria for oscillation of (2.12) and (2.13), we immediately obtain criteria for oscillation of (1.1).

Corollary 2.11. Assume that there exists a function $\zeta(t) \in C^{\prime}\left(\left[t_{0}, \infty\right)\right)$ such that (2.11) holds. If $\alpha=\beta$,

$$
\liminf _{t \rightarrow \infty} \int_{\sigma(t)}^{t} Q_{1}(s) d s>\frac{1}{e}
$$


and

$$
\liminf _{t \rightarrow \infty} \int_{\tau(t)}^{t} Q_{2}(s) d s>\frac{1}{e}
$$

then (1.1) is oscillatory.

Proof. The proof follows from Theorem 2.1.1 in [18] and Theorem 2.10. This completes the proof.

Corollary 2.12. Assume that there exists a function $\zeta(t) \in C^{\prime}\left(\left[t_{0}, \infty\right)\right)$ such that (2.11) holds. If $\alpha>\beta$,

$$
\int_{t_{1}}^{\infty} Q_{1}(s) d s=\infty
$$

and

$$
\int_{t_{1}}^{\infty} Q_{2}(s) d s=\infty
$$

for all $t_{1} \geq t_{0}$, then (1.1) is oscillatory.

Proof. The proof follows from Theorem 3.9.3 of [18] and Theorem 2.10. This completes the proof.

Corollary 2.13. Assume that there exists a function $\zeta(t) \in C^{\prime}\left(\left[t_{0}, \infty\right)\right)$ such that (2.11) holds. Suppose $\beta>\alpha, \theta_{1} \in$ $(0,1), \theta_{2}(0,1), \sigma(t)=\theta_{1} t, \tau(t)=\theta_{2} t$, If there exist

$$
\lambda_{1}>-\ln (\beta / \alpha) / \ln \left(\theta_{1}\right), \quad \lambda_{2}>-\ln (\beta / \alpha) / \ln \left(\theta_{2}\right)
$$

such that

$$
\liminf _{t \rightarrow \infty}\left(\theta_{1}(t) \exp \left(-t^{\lambda_{1}}\right)\right)>0
$$

and

$$
\liminf _{t \rightarrow \infty}\left(\theta_{2}(t) \exp \left(-t^{\lambda_{2}}\right)\right)>0
$$

hold, then (1.1) is oscillatory.

Corollary 2.14. Assume that there exists a function $\zeta(t) \in C^{\prime}\left(\left[t_{0}, \infty\right)\right)$ such that (2.11) holds. Suppose $\beta>\alpha, \theta_{1} \in$ $(0,1), \theta_{2}(0,1), \sigma(t)=t^{\theta_{1}}, \tau(t)=t^{\theta_{2}}$, If there exist

$$
\lambda_{1}>-\ln (\beta / \alpha) / \ln \left(\theta_{1}\right), \quad \lambda_{2}>-\ln (\beta / \alpha) / \ln \left(\theta_{2}\right)
$$

such that

$$
\liminf _{t \rightarrow \infty}\left(\theta_{1}(t) \exp \left(-(\ln t)^{\lambda_{1}}\right)\right)>0
$$

and

$$
\liminf _{t \rightarrow \infty}\left(\theta_{2}(t) \exp \left(-(\ln t)^{\lambda_{2}}\right)\right)>0
$$

hold, then (1.1) is oscillatory.

The proof of the Corollaries 2.12 and 2.13 follow from Theorem 4 and Theorem 5 of [11] and Theorem 2.10 respectively.

Our final result is concerned with the case when

$$
\int_{t_{0}}^{\infty} F(t) d t<\infty .
$$


Theorem 2.15. Let $\alpha=\beta$ and (2.6) hold. If (2.18) and

$$
\limsup _{t \rightarrow \infty} \eta^{\alpha}(\sigma(t)) \int_{t}^{\infty} F(s) d s>1,
$$

then (1.1) is oscillatory.

Proof. Let $y(t)$ be an eventually positive solution of (1.1). Then by Corollary 2.3, $y(t)$ is also a positive solution of (2.3) and so either $y(t) \in \mathcal{N}_{0}$ or $y(t) \in \mathcal{N}_{2}$ for all $t \geq t_{1} \geq t_{0}$. First assume that $y(t) \in \mathcal{N}_{2}$. Define

$$
w(t)=\frac{d(t)\left(c(t)\left(y^{\prime}(t)\right)^{\alpha}\right)^{\prime}}{y^{\alpha}(\sigma(t))}, t \geq t_{1} .
$$

Then $w(t)>0$ and using $(2.3)$ and $\left(H_{3}\right)$, we see that

$$
\begin{aligned}
w^{\prime}(t) & =-F(t)-\frac{\alpha d(t)\left(c(t)\left(y^{\prime}(t)\right)^{\alpha}\right)^{\prime}}{y^{\alpha+1}(\sigma(t))} y^{\prime}(\sigma(t)) \sigma^{\prime}(t) \\
& \leq-F(t) .
\end{aligned}
$$

Integrating the last inequality from $t$ to $\infty$, we obtain

$$
\int_{t}^{\infty} F(s) d s \leq \frac{d(t)\left(c(t)\left(y^{\prime}(t)\right)^{\alpha}\right)^{\prime}}{y^{\alpha}(\sigma(t))} .
$$

Using (2.4) in the above inequality yields

$$
\eta^{\alpha}(\sigma(t)) \int_{t}^{\infty} F(s) d s \leq 1
$$

which contradicts (2.19) as $t \rightarrow \infty$. The proof for the case $y(t) \in \mathcal{N}_{0}$ is similar to that of Theorem 2.6. The proof is now completed.

\section{Examples}

In this section, we present two examples to illustrate our main results.

Example 3.1. Consider the third-order nonlinear delay differential equation

$$
\left(t^{2}\left(\frac{1}{t}\left(y^{\prime}(t)\right)^{\frac{1}{3}}\right)^{\prime}\right)^{\prime}+\lambda t y^{\frac{1}{3}}(t / 2)=0, t \geq 1 .
$$

Compared to (1.1), we see that $b(t)=t^{2}, a(t)=\frac{1}{t}, f(t)=\lambda t, \lambda>0, \sigma(t)=\frac{t}{2}$ and $\alpha=\beta=\frac{1}{3}$. Clearly (3.1) is semi-canonical. A simple calculation shows that $\Pi(t)=\frac{1}{t}, d(t)=c(t)=1, F(t)=\lambda$. The transformed equation

$$
\left(\left(y^{\prime}(t)\right)^{\frac{1}{3}}\right)^{\prime \prime}+\lambda y^{\frac{1}{3}}\left(\frac{t}{2}\right)=0, t \geq 1
$$

is clearly canonical since the condition (2.1) is satisfied. Now the conditions (2.6) and (2.7) become

$$
\limsup _{t \rightarrow \infty} \int_{t / 2}^{t}\left(\int_{s}^{t} \int_{u}^{t} \lambda d v d u\right)^{3} d s=\infty>1
$$

and

$$
\liminf _{t \rightarrow \infty} \int_{t / 2}^{t} \frac{\lambda}{4}\left(\frac{s}{2}-1\right)^{\frac{4}{3}} d s=\infty>1 / e,
$$


that is, (2.6) and (2.7) are satisfied. Hence by Theorem 2.8 we see that (3.1) is oscillatory.

Note that the existing results reported in $[4,10,12,19,20,25]$ cannot be applied to equation(3.1) since $b(t) \neq 1$ and $\alpha \neq 1$.

Example 3.2. Consider the third-order linear delay differential equation

$$
\left(t^{2}\left(\frac{1}{t} y^{\prime}(t)\right)^{\prime}\right)^{\prime}+\frac{\lambda}{\sqrt{t}} y\left(\frac{t}{2}\right)=0, t \geq 1
$$

Compared to (1.1), we see that $b(t)=t^{2}, a(t)=\frac{1}{t}, f(t)=\frac{\lambda}{\sqrt{t}}, \lambda>0, \sigma(t)=\frac{t}{2}$ and $\alpha=\beta=1$. Clearly (3.3) is semi-canonical. A simple calculation shows that $\Pi(t)=\frac{1}{t}, d(t)=c(t)=1, F(t)=\frac{\lambda}{t^{\frac{3}{2}}}$ and $\eta(t)=\frac{t^{2}}{4}-t+\frac{3}{4}$. It is easy to see that the transformed equation

$$
y^{\prime \prime \prime}(t)+\frac{\lambda}{t^{2}} y(t / 2)=0, t \geq 1
$$

is canonical. Also the condition (2.1) and (2.18) are clearly hold. Now the conditions (2.6) and (2.19) become

$$
\limsup _{t \rightarrow \infty} \int_{t / 2}^{t}\left(\int_{s}^{t} \int_{u}^{t} \frac{\lambda}{v^{3 / 2}} d v d u\right) d s=\lim _{t \rightarrow \infty}\left(\sqrt{2}-\frac{11}{12}\right) t^{3 / 2}>1
$$

and

$$
\limsup _{t \rightarrow \infty}\left(\frac{t^{2}}{4}-t+\frac{3}{4}\right) \int_{t}^{\infty} \frac{\lambda}{s^{3 / 2}} d s=\lim _{t \rightarrow \infty} \lambda\left(\frac{t^{3 / 2}}{4}-\sqrt{t}+\frac{3}{4 \sqrt{t}}\right)=\infty,
$$

that is, (2.6) and (2.15) are satisfied. Hence by Theorem 2.15, the equation (3.3) is oscillatory.

Note that by applying Corollary 1 of [4], we see that equation (3.2) is oscillatory. However, the existing results presented in $[10,12,19,20,25]$ cannot be applied to equation (3.2) since $b(t) \neq 1$.

\section{Conclusion}

In this paper, we have introduced a new technique that transform the semi-canonical equation (1.1) into canonical equation. In this way, one can require less number of conditions to establish oscillation criteria for (1.1) which are significantly different from those reported in $[4,10,12,19,20,25]$. Further examples are provided to illustrate the importance of the main results.

Acknowledgements: The authors would like to express their gratitude to the editor and the anonymous referee for the careful reading of the original manuscript and useful comments that helped to improve the presentation of the results.

Conflict of interest: The authors declare that they have no conflict of interest.

Data Availability Statement: Data sharing is not applicable to this article as no datasets were generated or analysed during the current study.

\section{References}

[1] R. P. Agarwal, M. F. Aktas and A. Tiryaki, On oscillation criteria for third-order nonlinear delay differential equations, Arch. Math.(Brno), 45 (2009), 1-18. 
[2] R. P. Agarwal, M. Bohner, T. Li and C. Zhang, A Philos-type theorem for third-order nonlinear retarded dynamic equations, Appl. Math. Comput., 249 (2014), 527-531.

[3] R. P. Agarwal, M. Bohner and W.T. Li, Nonoscillation and Oscillation Theory for Functional Differential Equations, Marcel Dekker, New York, 2004.

[4] R. P. Agarwal, M. Bohner, T. Li and C. Zhang, Oscillations of third-order nonlinear delay differential equations, Taiwanese J. Math., 17 (2013), 545-558.

[5] R. P. Agarwal, S.R. Grace and T. Smith, Oscillation of certain third-order functional differential equations, Adv. Math. Sci. Appl., 16 (2006), 67-94.

[6] M. F. Aktas, A. Tiryaki and A. Zafer, Integral criteria for oscillation of third-order nonlinear differential equations, Nonlinear Anal., 71 (2009), 1496-1502.

[7] M. Aktas, A. Tiryaki and A. Zafer, Oscillation criteria for third-order nonlinear functional differential equations, Appl. Math. Lett., 23 (2010), 756-762.

[8] B. Baculikova, Asymptotic properties of noncanonical third-order differential equations, Math. Slovaca, 69 (2019), 13411350.

[9] B.Baculikova and J. Dzurina, Oscillation of third-order nonlinear differential equations, Appl. Math. Lett., 24(2011), $466-470$.

[10] B. Baculikova and J. Dzurina, Oscillation of third-order functional differential equations, Electron. J. Qual. Theory Differ. Equ., 43, $1(2010)$.

[11] B. Baculikova, J. Dzurina and Y. V. Ragovchenko, Oscillation of third-order trinomial delay diferential equations. Appl. Math. Comput., 218 (2012), 7023-7033.

[12] G. E. Chatzarakis, J. Dzurina and I. Jadlovska, Oscillation and asymptotic properties of third-order quasilinear delay differential equations, J. Inequ. Appl., (2019) 2019:23, pp.17.

[13] G. E. Chatzarakis, S. R. Grace and I. Jadlovska, Oscillation criteria for third-order delay differential equations, Adv. Differece Equ., (2017) 2017: 330, pp.11.

[14] J. Dzurina, Asymptotic properties of the third-order delay differential equations, Nonlinear Anal., 26(1996), 33-39.

[15] J. Dzurina and I. Jadlovska, Oscillation of third-order differential equations with noncanonical operators, Appl. Math. Comput., 336 (2018), 394-402.

[16] E. M. Elabbasy, T. S. Hassan and B. M. Elmatary, Oscillation criteria for third-order delay nonlinear differential equations, Electron. J. Qual. Theory Diff. Equ., 5, 11 (2012).

[17] L. H. Erbe, Q. Kong and B. G. Zhang, Oscillation Theory for Functional Differential Equations, Marcel Dekker, New York, 1995.

[18] I. Gao, Q. Zhang and S. Liu, Oscillation behavior of third-order nonlinear delay differential equations on time scales, J. Comput. Appl. Math., 256 (2014), 104-113.

[19] S. R. Grace, R. P. Agarwal and M. F. Aktas, On the oscillation of third-order functional differential equations, Indian J. Pure Appl. Math., 39 (2008), 491-507.

[20] S. R. Grace, R.P.Agarwal, R. Pavani and E. Thandapani, On the oscillation of certain third-order nonlinear functional differential equations, Appl. Math. Comput., 202(2008), 102-112.

[21] J. R. Graef and S. H. Saker, Oscillation theory of third-order nonlinear functional differential equations, Hiroshima Math. J., 43 (2013), 49-72.

[22] G. Jayaraman, N. Padmanabhan and R. Mohrotra, Entry flow into a circular tube slowly varying cross section, Fluid Dyn. Res, 1 (1986), 131-144.

[23] I. T. Kiguradze and T. A. Chanturia, Asymptotic Properties of Solutions of Nonautonomous Ordinary Differential Equations, Kluwer Acad. Publ., Dordrech, 1993.

[24] T. Kusano and M. Naito, Comparison theorems for functional differential equations with deviating arguments, J. Math. Soc. Japan, 3 (1981),509-533.

[25] T. Li, C. Zhang, B. Baculikova and J. Dzurina, On the oscillation of third-order quasi-linear delay differential equations, Tatra Mt. Math. Publ., 48 (2011), 117-123.

[26] H. P. McKean, Nagumo's equation, Advances in Math., 4 (1970), 209-223.

[27] S. Pathi and S. Pati, Theory of Third-Order Differential Equations, Springer, New-Delhi, 2014.

[28] Ch. G. Philos, On the existence of nonoscillatory solutions tending to zero at $\infty$ for differential equations with positive delay, Arch. Math.(Basel), 36 (1981), 168-178.

[29] S. H. Saker, Oscillation criteria of third-order nonlinear delay differential equations, Math. Solvaca, 56 (2006), 433-456.

[30] S. H. Saker and J. Dzurina, On the oscillation of certain class of third-order nonlinear delay differential equations, Math. Bohem., 135 (2010), 225-237.

[31] X. H. Tang, Oscillation for first order superlinear delay differential equations, J. Londan Math. Soc., 65 (2002), 115-122.

[32] W.F. Trench, Canonical forms and principal systems for general disconjugate equations, Trans. Amer. Math. Soc., 184 (1974), 319-327.

[33] S. D. Vreeke and G. M. Sandquist, Phase space analysis of reactor kinetics, Nucl. Sci. Eng., 42 (1970), 259-305.

[34] C. Zhang, T. Li, B. Sun and E. Thandapani, On the oscillation of higher order half-linear delay differential equations, Appl. Math. Lett., 24 (2011), 1618-1621. 